\title{
OPTICAL RADIATION FROM CHANNELED RELATIVISTIC HEAVY IONS IN VICINITY OF THE CHERENKOV ANGLE
}

\author{
Elena Fiks $^{a, 1}$, Oleg Bogdanov ${ }^{a}$, Yury Pivovarov ${ }^{b}$ \\ ${ }^{a}$ National Research Tomsk Polytechnic University, Tomsk, Russia \\ ${ }^{b}$ V.E.Zuev Institute of Atmospheric Optics, Tomsk, Russia
}

The Cherenkov radiation (ChR) from relativistic charged particles moving uniformly and rectilinearly with constant velocity in an optically transparent radiator is described by the well-known Tamm-Frank theory. Several reasons may lead to deviations from this ideal character of motion: 1) multiple scattering - a particle velocity vector changes and angular distribution becomes broadened; 2) stopping in a radiator - a particle velocity decreases, the Cherenkov angle changes; 3) channeling effect. Multiple scattering and channeling are concurrent processes and in a thin crystal the deviation from rectilinear trajectory is mainly due to periodic transverse oscillations between crystallographic planes. Here, the ChR from channelled RHI in an optically transparent diamond crystal is investigated in detail. We analyze: a) the shift of both ChR spectral and angular distributions maxima compared to the standard Tamm-Frank theory due to taking account of periodic deviation of the channelled RHI velocity vector from the average one; b) dependence of the ChR intensity on the azimuthal angle; c) influence of slowing-down due to ionization energy loss. The effects predicted strongly depend on the channelled RHI energy and are closely connected to the normal and anomalous Doppler effects in emission from an oscillator moving in the medium.

1 Corresponding author: elenafiks@gmail.com 